\title{
Cost allocation protocols for network formation on connection situations
}

\author{
B. Escoffier, L. Gourvès, J. Monnot, S. Moretti
}

CNRS UMR 7243

Laboratoire d'Analyse et Modèlisation de Systèmes pour l'Aide à la DEcision (Lamsade)

Paris Dauphine University

ValueTools 2012

8-12 October 2012 Cargese, France 


\section{Summary}

Model and objectives

Game and properties

An optimal protocol 


\section{Building a network in a strategic setting}

Situation: a set of agents building a network in order to be connected to a given source.

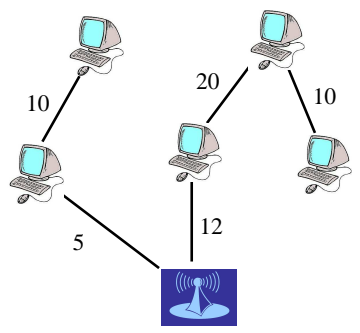

Assumptions:

- making a link $e=(i, j)$ has a cost/consumes energy $w(e)$;

- non cooperative game: agents do not make binding agreements on the design of the network. Each agent wants to minimize its own cost. 


\section{Question and objectives}

How should we design cost allocation protocols to minimize the efficiency loss caused by rational players that are only willing to perform update leading to an immediate reduction of their individual cost shares? 


\section{Question and objectives}

How should we design cost allocation protocols to minimize the efficiency loss caused by rational players that are only willing to perform update leading to an immediate reduction of their individual cost shares?

$\rightarrow$ address the problem of the design of cost allocation protocols to coordinate players placed on the nodes of a graph in such a way that:

- convergence under Better Response Dynamics (BRD) holds

- an efficient (minimum cost) communication network is built. 


\section{The strategic game}

- $G=\left(N^{\prime}, E, w\right)$ is an undirected, connected and weighted graph, where $N^{\prime}=N \cup\{0\}$ and $N=\{1,2, \ldots, n\}$, and $w(e)$ is the cost/power needed to build/use the link $e$. 


\section{The strategic game}

- $G=\left(N^{\prime}, E, w\right)$ is an undirected, connected and weighted graph, where $N^{\prime}=N \cup\{0\}$ and $N=\{1,2, \ldots, n\}$, and $w(e)$ is the cost/power needed to build/use the link $e$.

- The strategy space $\mathcal{N}_{G}(i)$ of every player $i \in N$ is his neighborhood in the graph. A state (or strategy profile) $S$ is a vector $\left(S_{1}, S_{2}, \cdots, S_{n}\right)$. 


\section{The strategic game}

- $G=\left(N^{\prime}, E, w\right)$ is an undirected, connected and weighted graph, where $N^{\prime}=N \cup\{0\}$ and $N=\{1,2, \ldots, n\}$, and $w(e)$ is the cost/power needed to build/use the link $e$.

- The strategy space $\mathcal{N}_{G}(i)$ of every player $i \in N$ is his neighborhood in the graph. A state (or strategy profile) $S$ is a vector $\left(S_{1}, S_{2}, \cdots, S_{n}\right)$.

- A protocol is a vector $\left(c_{1}(G, S), \cdots, c_{n}(G, S)\right)$ which, given a graph $G$ and a strategy profile $S$, allocates a cost to the players. 


\section{The strategic game}

- $G=\left(N^{\prime}, E, w\right)$ is an undirected, connected and weighted graph, where $N^{\prime}=N \cup\{0\}$ and $N=\{1,2, \ldots, n\}$, and $w(e)$ is the cost/power needed to build/use the link $e$.

- The strategy space $\mathcal{N}_{G}(i)$ of every player $i \in N$ is his neighborhood in the graph. A state (or strategy profile) $S$ is a vector $\left(S_{1}, S_{2}, \cdots, S_{n}\right)$.

- A protocol is a vector $\left(c_{1}(G, S), \cdots, c_{n}(G, S)\right)$ which, given a graph $G$ and a strategy profile $S$, allocates a cost to the players.

- The cost of remaining disconnected from the source is infinite. 
An example of game form with two players
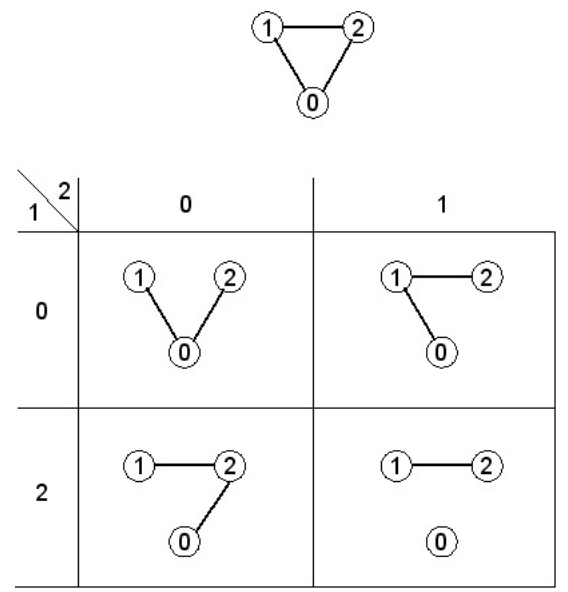


\section{Some properties for protocols and games}

- A cost allocation protocol $c$ such that $\sum_{i \in S} c_{i}(w, S)=w\left(T_{S}\right)$ for every strategy profile $S$ is said budget-balanced.

(for connected players if some players are not connected)

( $T_{S}=\left\{\left(i, S_{i}\right): i \in S\right\}$ is the network of (connected) players under state $S)$ 


\section{Some properties for protocols and games}

- A cost allocation protocol $c$ such that $\sum_{i \in S} c_{i}(w, S)=w\left(T_{S}\right)$ for every strategy profile $S$ is said budget-balanced. (for connected players if some players are not connected) ( $T_{S}=\left\{\left(i, S_{i}\right): i \in S\right\}$ is the network of (connected) players under state $S)$

A protocol is said state-dependent iff for every state $S$ and every weight functions $w, w^{\prime}$, with $w(e)=w^{\prime}(e)$ for every $e \in T_{S}$, then $c_{i}(w, S)=c_{i}\left(w^{\prime}, S\right)$ for every $i \in S$. 
The Bird protocol

Each player $i$ pays the cost of the link from him to the its predecessor on the unique path from the source 0 to $i$. 


\section{The Bird protocol}

Each player $i$ pays the cost of the link from him to the its predecessor on the unique path from the source 0 to $i$.
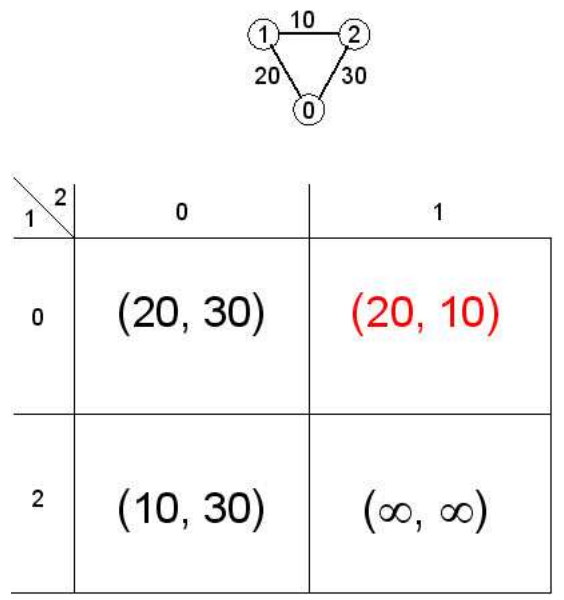


\section{The Bird protocol}

Each player $i$ pays the cost of the link from him to the its predecessor on the unique path from the source 0 to $i$.
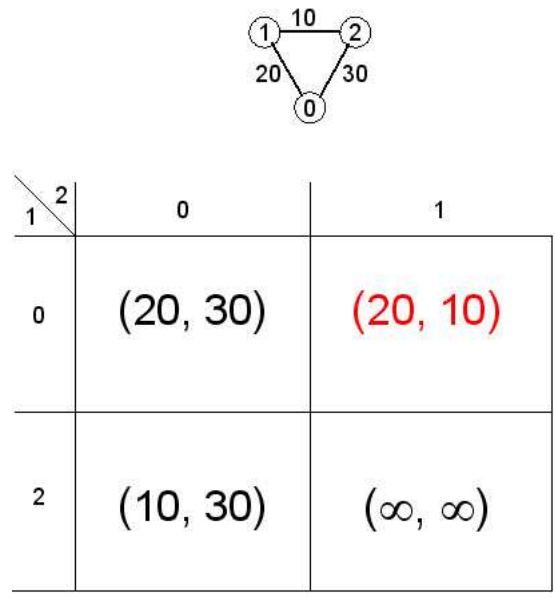

Nash equilibrium $(0,1)$ is optimal (i.e. $T_{(0,1)}$ is a minimum cost spanning tree (mcst) connecting all nodes in $\left.N^{\prime}\right)$, but $(2,0)$ is not. 


\section{Better Response Dynamic and Optimality}

- Given a protocol $c$, a strategy $x \in \mathcal{N}_{G}(i)$ is a better response of player $i$ with respect to the strategy profile $S$ if $c_{i}\left(G,\left(x, S_{-i}\right)\right)<c_{i}(G, S)$. 


\section{Better Response Dynamic and Optimality}

- Given a protocol $c$, a strategy $x \in \mathcal{N}_{G}(i)$ is a better response of player $i$ with respect to the strategy profile $S$ if $c_{i}\left(G,\left(x, S_{-i}\right)\right)<c_{i}(G, S)$.

- A Better Response Dynamic (BRD, also called Nash dynamics) (associated with a protocol $c$ ) is a sequence of states $S^{0}, S^{1}, \ldots$, such that each state $S^{k}$ (except $S^{0}$ ) is resulted by a better response of some player from the state $S^{k-1}$. 


\section{Better Response Dynamic and Optimality}

- Given a protocol $c$, a strategy $x \in \mathcal{N}_{G}(i)$ is a better response of player $i$ with respect to the strategy profile $S$ if $c_{i}\left(G,\left(x, S_{-i}\right)\right)<c_{i}(G, S)$.

- A Better Response Dynamic (BRD, also called Nash dynamics) (associated with a protocol $c$ ) is a sequence of states $S^{0}, S^{1}, \ldots$, such that each state $S^{k}$ (except $S^{0}$ ) is resulted by a better response of some player from the state $S^{k-1}$.

- We say that a cost allocation protocol is optimal iff every associated BRD reaches an optimum Nash equilibrium (mcst). 


\section{Better Response Dynamic and Optimality}

- Given a protocol $c$, a strategy $x \in \mathcal{N}_{G}(i)$ is a better response of player $i$ with respect to the strategy profile $S$ if $c_{i}\left(G,\left(x, S_{-i}\right)\right)<c_{i}(G, S)$.

- A Better Response Dynamic (BRD, also called Nash dynamics) (associated with a protocol $c$ ) is a sequence of states $S^{0}, S^{1}, \ldots$, such that each state $S^{k}$ (except $S^{0}$ ) is resulted by a better response of some player from the state $S^{k-1}$.

- We say that a cost allocation protocol is optimal iff every associated BRD reaches an optimum Nash equilibrium (mcst).

Theorem If a protocol is budget-balanced and optimal, then it is not state-dependent. 


\section{A budget-balanced and optimal protocol}

Idea: If the network is not optimal (extra cost $\Delta$ ), charge this cost $\Delta$ to a player (a set of players) to create for them an incentive to change.

Problem: find who should pay!

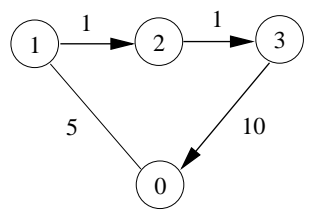




\section{A budget-balanced and optimal protocol}

Idea: If the network is not optimal (extra cost $\Delta$ ), charge this cost $\Delta$ to a player (a set of players) to create for them an incentive to change.

Problem: find who should pay!
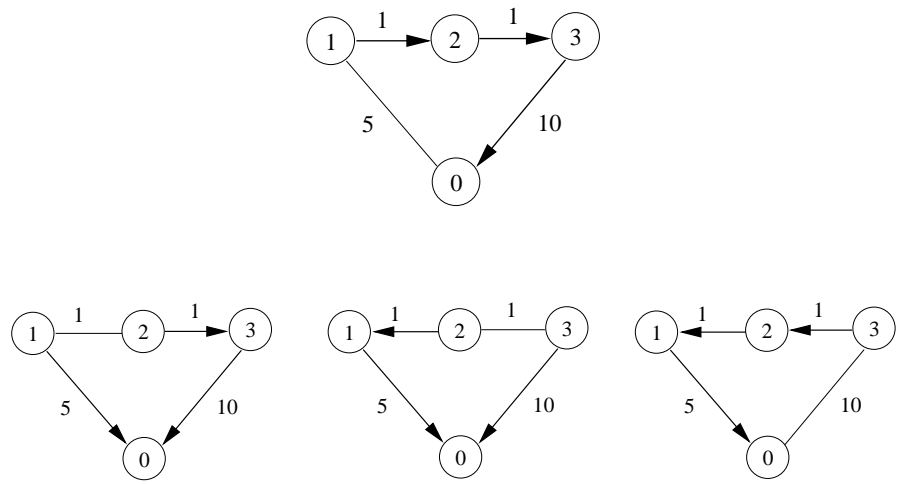


\section{A budget-balanced and optimal protocol (2)}

How to find the victims: based on a set of players which

(1) do not play as we want (as in a given mcst)

(2) have additional properties on the connectivity of players. 


\section{A budget-balanced and optimal protocol (2)}

How to find the victims: based on a set of players which

(1) do not play as we want (as in a given mcst)

(2) have additional properties on the connectivity of players.

Theorem A state $S$ corresponds to a mcst if and only if it is a Nash equilibrium. 


\section{A budget-balanced and optimal protocol (2)}

How to find the victims: based on a set of players which

(1) do not play as we want (as in a given mcst)

(2) have additional properties on the connectivity of players.

Theorem A state $S$ corresponds to a mcst if and only if it is a Nash equilibrium.

Two protocols:

- One fairly (equally) shares cost between players in a optimal situation;

- One is more like Bird's protocol (players pay one link). 


\section{Ordinal potential game}

Theorem BRD always converges after at most $m n^{2}$ rounds, where $m$ is the number of edges of the graph. 


\section{Ordinal potential game}

Theorem BRD always converges after at most $m n^{2}$ rounds, where $m$ is the number of edges of the graph.

Thanks to a potential function $\Phi(S)$, ie $\Phi$ only decreases during a BRD.

$\Phi(S)=\left(|N \backslash \operatorname{con}(S)|,|\hat{V}(S)|, \sum_{i \in \hat{V}(S)}\left|E_{S}(i)\right|\right)$

where $E_{S}(i)=\left\{j \in N^{\prime}: w(i, j)<w\left(i, S_{i}\right)\right\}$. 


\section{Conclusions}

- We have studied cost allocation protocols for connection situations in a strategic setting. 


\section{Conclusions}

- We have studied cost allocation protocols for connection situations in a strategic setting.

- we have analyzed properties for protocols in relation the the convergence of the best reply dynamics to efficient Nash equilibria. 


\section{Conclusions}

- We have studied cost allocation protocols for connection situations in a strategic setting.

- we have analyzed properties for protocols in relation the the convergence of the best reply dynamics to efficient Nash equilibria.

- the inherent limitations of the optimal protocols proposed in this paper is that it depends on the choice of an a priori selected mcst. 